\title{
Efficacy and Safety of Neurokinin-1 Receptor Antagonists for Prevention of Chemotherapy-Induced Nausea and Vomiting: Systematic Review and Meta-analysis of Randomized Controlled Trials
}

\author{
Dong-Mei Yuan ${ }^{1 \&}$, Qian $\mathrm{Li}^{1 \&}$, Qin Zhang ${ }^{2}$, Xin-Wu Xiao', Yan-Wen Yao ${ }^{1}$, Yan \\ Zhang ${ }^{1}$, Yan-Ling $\mathrm{Lv}^{3}$, Hong-Bin Liu ${ }^{1}$, Tang-Feng Lv ${ }^{1}$, Yong Song ${ }^{1 *}$
}

\begin{abstract}
Objectives: Can addition of neurokinin-1 receptor antagonists (NK1-RAs) be considered as an ideal strategy for the prevention of chemotherapy-induced nausea and vomiting (CINV)? Researchers differ on this question. Materials and Methods: Electronic databases were searched for randomized control trials (RCTs) that evaluated the effectiveness and safety of NK1-RAs in preventing CINV. The primary end point was complete response (CR) in the acute, delayed, and overall phases after chemotherapy. Subgroup analyses evaluated the types of NK1-RAs, routines of administration, types of malignancies, regimens used in combination with NK1-RAs, and age of patients included in the studies. The incidences of different types of adverse events were also extracted to estimate the safety of NK1-RAs. Results: A total of 38 RCTs involving 13,923 patients were identified. The CR rate of patients receiving NK-RAs was significantly higher than patients in the control groups during overall phase $(70.8 \%$ vs $56.0 \%, P<0.001)$, acute phase $(85.1 \%$ vs $79.6 \%, P<0.001)$, and delayed phase $(71.4 \%$ vs $58.2 \%$, $P<0.001)$. There were three studies including patients of children or adolescents, the $C R$ rate was also significantly higher in the treatment group (overall phase: $O R=2.807, P<0.001$; acute phase: $O R=2.863, P=0.012$; delayed phase: $\mathrm{OR}=2.417, P<0.001$ ). For all the other outcomes, patients in the NK1-RAs groups showed improvements compared to the control groups (incidence of nausea: $45.2 \%$ vs $45.9 \%, P<0.001$; occurrence of vomiting: $22.6 \%$ vs $38.9 \%, P<0.001$; usage of rescue drugs: $23.5 \%$ vs $34.1 \%, P<0.001)$. The pooled side effects from NK1-RAs did not significantly differ from previous reports and the toxicity rates in patients less than eighteen years old also did not diff between the two groups $(P=0.497)$. However, we found that constipation and insomnia were more common in the patients of control groups, whereas diarrhea and hiccups were more frequently detected in patients receiving NK1-RAs. Conclusions: NK1-RAs improved the CR rate of CINV. They are effective for both adults and children. The use of NK1-RAs might be associated with the appearance of diarrhea and hiccups, while decreasing the possibility of constipation and insomnia.
\end{abstract}

Keywords: Neurokinin-1 receptor antagonist - chemotherapy-induced nausea and vomiting - aprepitant - meta-analysis

Asian Pac J Cancer Prev, 17 (4), 1661-1675

\section{Introduction}

Chemotherapy-induced nausea and vomiting (CINV) has a significant adverse effect on health-related quality of life and even has negative impacts on the continuation of chemotherapy(Martin et al., 2003a; Martin et al., 2003b). Less toxic chemotherapy is important to retain good performance status and enable further treatment of cancer patients, and prevention of CINV remains the most important issue in supportive therapy of cancer patients.

According to the frequency and power of emetic action, chemotherapy agents are categorized into a four- level classification scheme: minimal $<10 \%$, low $10-30 \%$, moderate $31-90 \%$, and high $>90 \%$ (Basch et al., 2011; Di Maio et al., 2013) Great advances have been made in controlling CINV during the past decade. However, nausea and vomiting remain to be significant problems for patients receiving highly or moderately emetogenic chemotherapy (HEC/MEC) (Grunberg et al., 2004; Bloechl-Daum et al., 2006). Meanwhile, CINV is classified into three categories: acute onset (occurring within 24 hours after initial administration of chemotherapy); delayed onset (occurring 24 hours to several days after chemotherapy treatment); and anticipatory nausea and vomiting (Aapro 
et al., 2005).

Dexamethasone plays a major role in the prevention of acute and delayed CINV and is an integral component of almost all anti-emetic regimens (Grunberg, 2007). And improvement quickly followed with the addition of 5-hydroxytryptamine (5HT3) receptor antagonists (RAs) (Hesketh, 2008; Saito et al., 2009). 5-HT3-RAs now form the cornerstone of the therapy for the control of acute emesis with MEC to HEC (Jordan et al., 2014). Navari et al firstly demonstrated that neurokinin-1 receptor antagonists (NK1-RAs) improve CINV when used in patients receiving cisplatin-based chemotherapy (Navari et al., 1999). NK1-RAs are thought to act centrally inhibiting emesis by blocking binding of substance $P$ at the NK1 receptor in the brain stem emetic center (Tattersall et al., 1996). Recent studies and guidelines recommend that the addition of NK1-RAs to the 5-HT3RAs plus corticosteroid combination as the most effective regimen for controlling both acute and delayed CINV (Aapro et al., 2015). Previous systematic review about the efficacy of NK1-RAs in the prevention of CINV was published in 2012 (dos Santos et al., 2012). dos Santos LV et al demonstrated that NK1-RAs increased CINV control in the acute, delayed, and overall phase, and NK1-RAs are effective for both HEC and MEC. However, further researches about NK1-RAs were still inconsistent. Roila $\mathrm{F}$ found that in cancer patients submitted to cisplatin-based chemotherapy, aprepitant plus dexamethasone was not superior to metoclopramide plus dexamethasone in preventing delayed emesis (complete response rate was $80.3 \%$ and $82.5 \%$, respectively) (Roila et al., 2015). Meanwhile, Kitayama et al reported that palanosetron and 1-day dexamethasone is almost equivalent to the combination fosaprepitant, granisetron and dexamethasone for MEC (Kitayama et al., 2015). To be followed, there were studies evaluated the efficacy of NK1-RAs in pediatric patients (Kang et al., 2015) and adolescent patients (Gore et al., 2009). Therefore, the aim of this study is to provide an updated systematic review of the efficacy and safety of NK1-RAs in the prevention of CINV, and to evaluate the use of NK1-RAs in pediatric and adolescent patients.

\section{Materials and Methods}

\section{Study searching}

Sources such as MEDLINE, EMBASE, the Cochrane Library database, ISI Web of Science were searched (last search, April 30th, 2015). We searched for randomized controlled trials (RCTs) that compared the addition of NK1-RAs to standard antiemetic regimens for cancer patients receiving chemotherapy. We used a combination of the following terms: neurokinin-1 receptor, aprepitant, fosaprepitant, netupitant, casopitant, chemotherapy induced nausea and vomiting. Furthermore, we manually searched the reference sections of the selected studies and relevant reviews for additional publications.

We included human studies written in English, and we did not restrict publication date. When the same patient population was used in several researches, only the most recent, largest or complete study was included.

\section{Inclusion criteria}

Two of the authors (YDM, LQ) independently established the eligibility of the studies retrieved from the databases and bibliographies. Trials were included in this meta-analysis if they met the following criteria: (i) published randomized controlled clinical trials with a parallel design comparing NK1-RAs alone or in combination with other antiemetic therapy to antiemetic therapy without NK1-RAs (placebo, dexamethasone, or 5-HT3-RAs); (ii) sufficient data on adequate description of outcomes or toxicity by different treatment; (iii) The studies were prospective RCTs. Disagreement between the two authors were resolved by discussion.

\section{Exclusion criteria}

Trials were excluded if they met any of the following criteria: (i) case reports, reviews and conference reports; (ii) studies based on overlapping cohorts from the same institutions.

\section{Data extraction and quality assessment}

The name of the first author and year of publication of the article were used for the purpose of identification. The following data were also extracted: study population (country where the study was conducted, Number of patients, type of cancer, type of chemotherapy), methodological characteristics of the RCTs (method of randomization, drop-out description), drugs used in research group and control group, types of NK1-RAs, and most common adverse events.

\section{Definitions of outcomes}

In the present research, outcomes were defined as follows: (i) the primary outcome that we extracted was the proportion of patients who achieved CR during the overall period of assessment (acute phase: $0-24 \mathrm{~h}$ after chemotherapy; delayed phase: 24 hours to several days after chemotherapy). CR was defined as the absence of vomiting and the absence of the need for rescue antiemetic therapy; (ii) the secondary outcomes were nausea, vomiting and need for rescue antiemetic therapy during the overall periods; (iii) safety and tolerability of the antiemetic regimens were also assessed. The most reported adverse events, such as constipation, neutropenia, hiccups, fatigue were also included in the meta-analysis as secondary outcomes.

\section{Subgroup analyses}

Predefined subgroup analyses were undertaken in clinically relevant subsets to evaluate the impact of these subgroups on the estimation of the effect size. The following comparisons were carried out: (i) different types of NK1-RAs (aprepitant vs casopitant vs fosaprepitant vs others); (ii) route of administration of NK1-RAs (oral vs intravenous vs both); (iii) age of included patients (adults vs children); (iv) different drugs used in the control arm (placebo vs others); (v) different types of malignancies included in the researches (solid tumor vs hematologic malignancy vs both); (vi) antiemetic regimens in combination with NK1-RAs in the research group (5-HT3RAs vs 5-HT3-RAs plus dexamethasone). Sensitivity 
analyses based on methodological quality parameters were performed to test for possible variations in estimates of overall OR between subgroups.

\section{Statistical analysis}

The Mantel-Haenszel random-effects method was used to calculate odds ratios (ORs) and the corresponding $95 \%$ confidence intervals (CIs) (DerSimonian and Laird, 1986). We considered an OR more than 1 favored the NK1-RAs group in the primary endpoints. And an OR more than 1 favored the controlled group in the secondary endpoints and adverse events.

For the test of heterogeneity, we used Higgins $\mathrm{I}^{2}$, which measures the percentage of total variation across trials (Higgins and Thompson, 2002). $\mathrm{I}^{2}$ ranges from 0 (no observed heterogeneity) to $100 \%$. Heterogeneity was considered substantial if $\mathrm{I}^{2}$ was equal to or more than $50 \%$. When heterogeneity was detected, a possible explanation for it was intensively pursued. If a reasonable cause was found, a separate analysis was then performed. If the cause was not apparent and if heterogeneity was generated by divergent data, the data would not be pooled.

Publication bias was assessed by using Begg's funnel plot and Egger's test. If publication bias existed, the Begg's funnel plot was asymmetric or the $P$ value was less than 0.05 by the Egger's test, the Trim and Fill method was subsequently used (Duval and Tweedie, 2000).

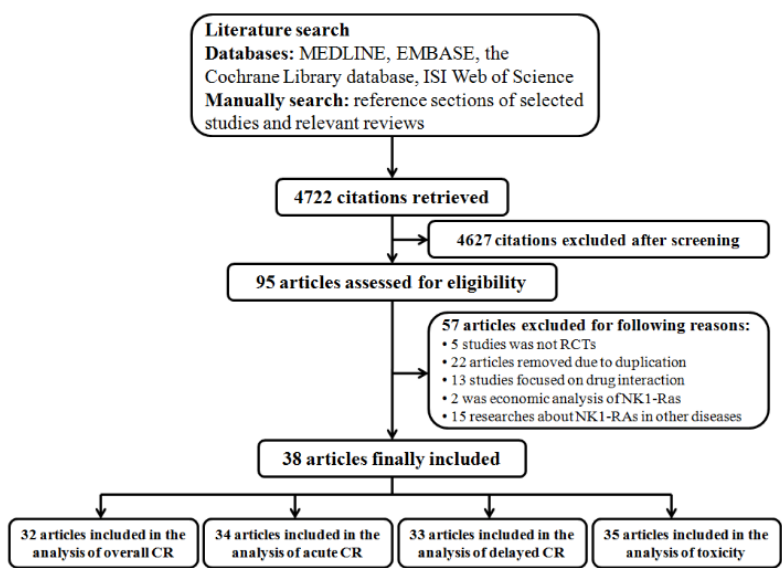

Figure 1. Flow chat of Identification and Selection of Studies
If a given study had more than one interventional arm, we chose to combine all intervention groups together to avoid multiple counting of the same individuals in the control arm (Altman and Bland, 1997). For example, if a trial had more than one research groups with different dose of NK1-RAs and on control arm without NK1-RAs (such as a research performed by Hesketh PJ et al (Hesketh et al., 2014)), the numbers of subjects in different research arms was added and then compared to control arm.

All the statistical tests used in our meta-analysis were performed with STATA version 11.0 software (Stata Corporation College Station, TX). A $P$-value $<0.05$ was considered statistically significant.

\section{Results}

\section{Literature search and study selection}

Figure 1 shows a flow diagram depicting how we identified the relevant clinical trials. By searching four databases and by hand-searching relevant bibliographies, a total of 4722 articles were identified (last search: April 30th, 2015). After screening of title and abstract, 95 studies that potentially met the inclusion criteria were closely scrutinized. 57 articles were further excluded for the following reasons: (i) 5 studies was not RCTs; (ii) 22 articles were removed because of duplication; (iii) 13 studies were about drug interaction; (iv) 2 articles were economic analyses of aprepitant-containing regimens; (v) 15 studies were about the application of NK1-RAs in diseases other than CINV.

There were 38 eligible RCTs, including 13923 patients in this analysis (Navari et al., 1999) (Hesketh et al., 1999; Campos et al., 2001; Cocquyt et al., 2001; Van Belle et al., 2002; Chawla et al., 2003; Hesketh et al., 2003; PoliBigelli et al., 2003; de Wit et al., 2004; Warr et al., 2005b; Schmoll et al., 2006; Herrington et al., 2008; Arpornwirat et al., 2009; Gore et al., 2009; Grunberg et al., 2009; Herrstedt et al., 2009; Roila et al., 2009; Yeo et al., 2009; Rapoport et al., 2010; Takahashi et al., 2010; Navari et al., 2011; Albany et al., 2012; Aksu et al., 2013; Saito et al., 2013; Stiff et al., 2013; Tanioka et al., 2013; Aapro et al., 2014; Hesketh et al., 2014; Hu et al., 2014; Ito et al., 2014; Roila et al., 2014; Schmitt et al., 2014; Badar et al.,
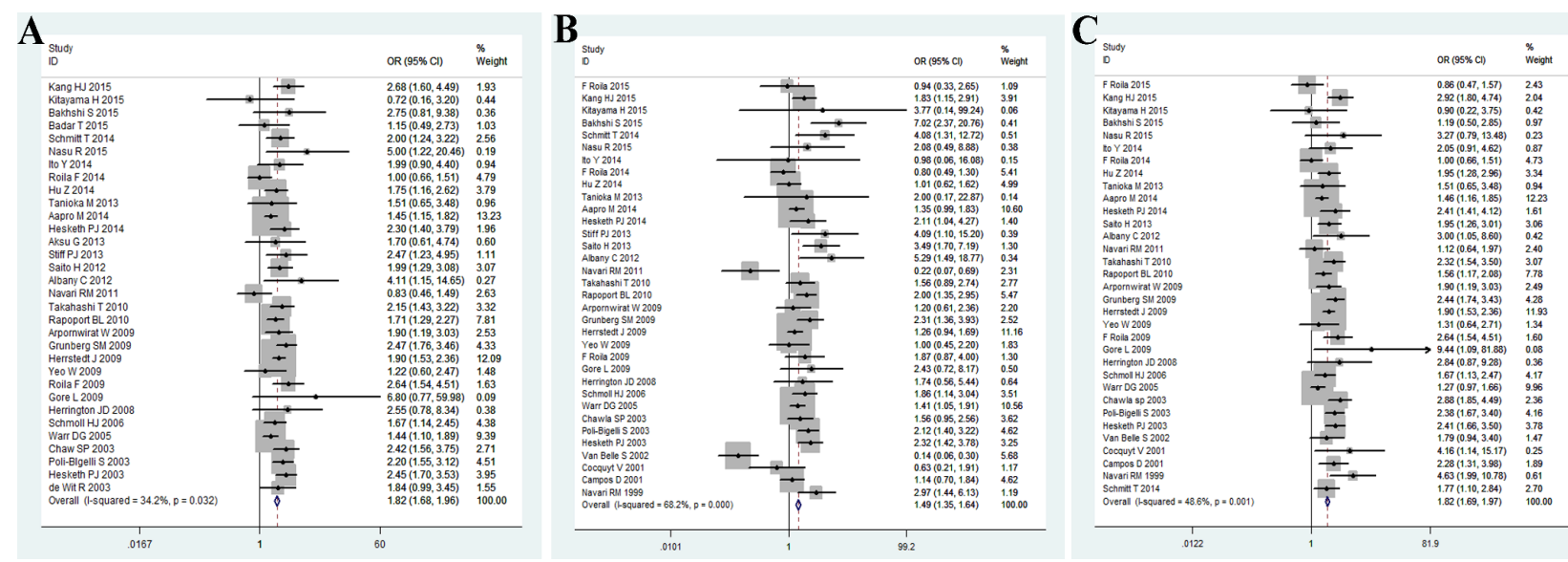

Figure 2. (A) Forest Plot of Odds Ratios for the Incidence of Overall Complete Response. (B) Summarized Odds Ratios for the Incidence of Complete Response in the Acute Phase. (C) Summarized Odds Ratios for the Incidence of Complete Response in the Delayed Phase 


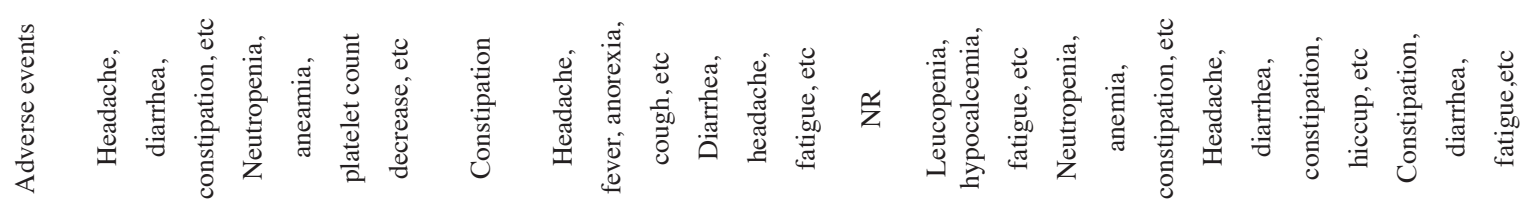

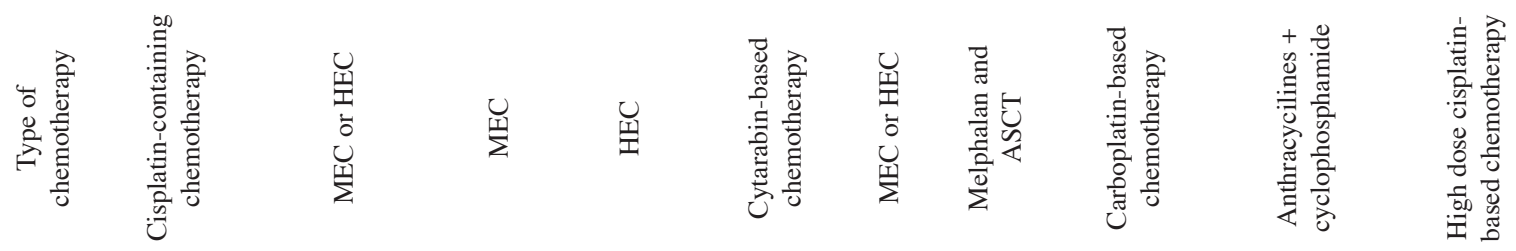

In

|| ||||||||

In:

\|\|\|\|\|\|\|\|

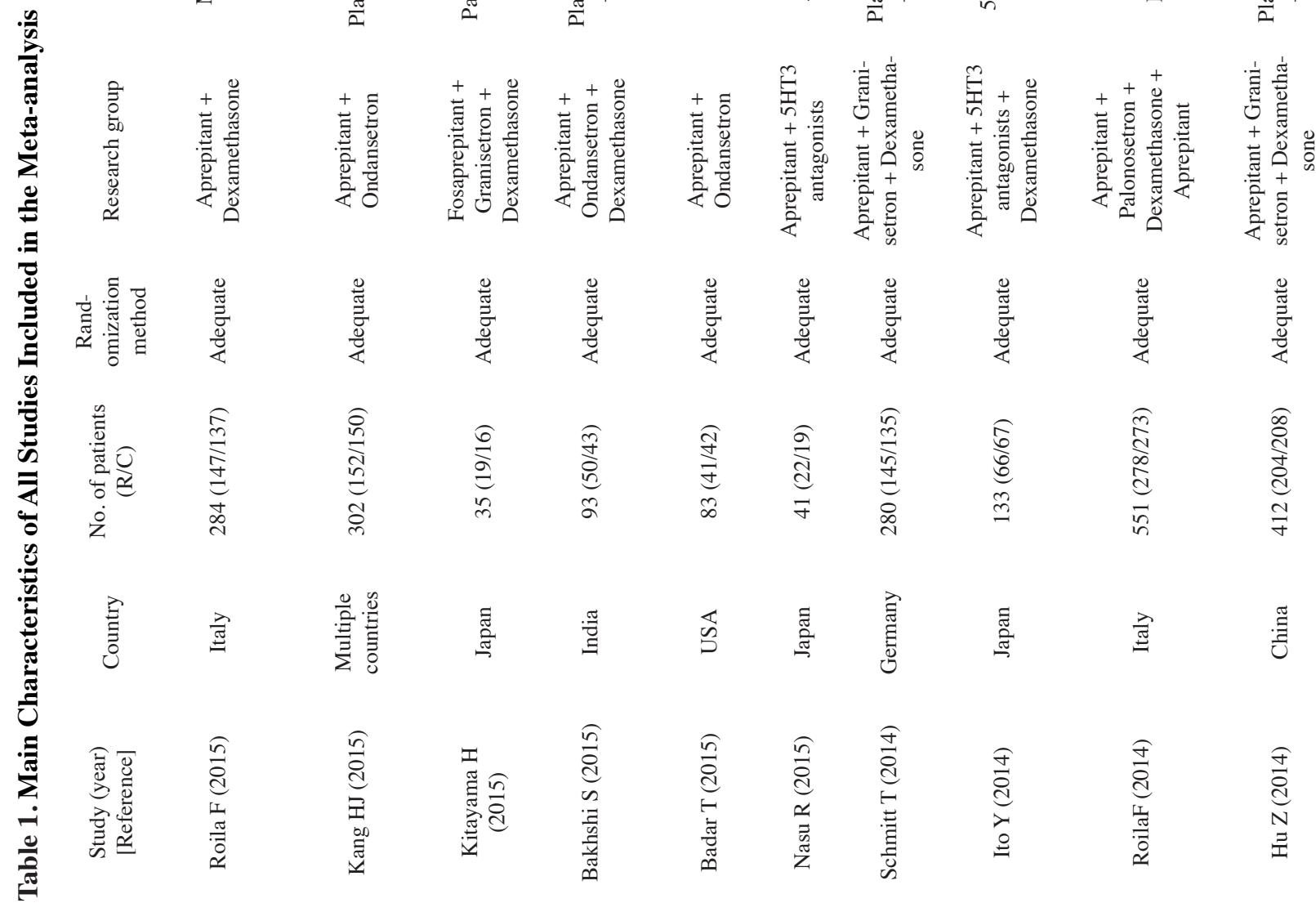


DOI:http://dx.doi.org/10.7314/APJCP.2016.17.4.1661 Neurokinin-1 Receptor Antagonists for Chemotherapy-Induced Nausea and Vomiting: Systematic Review

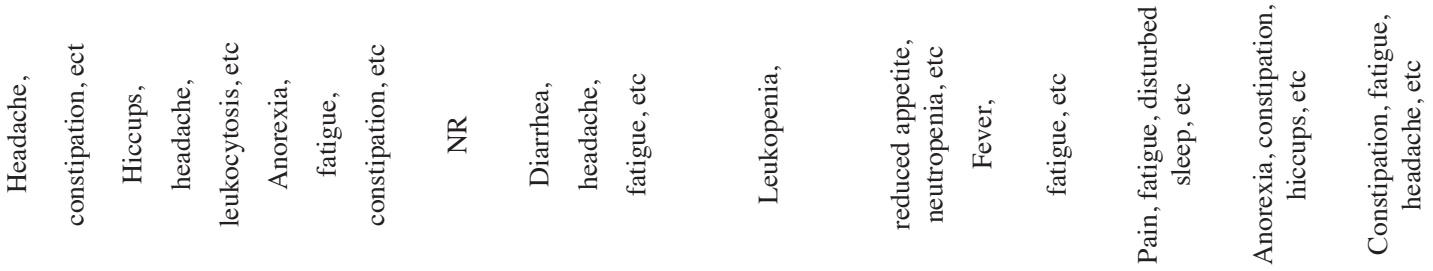

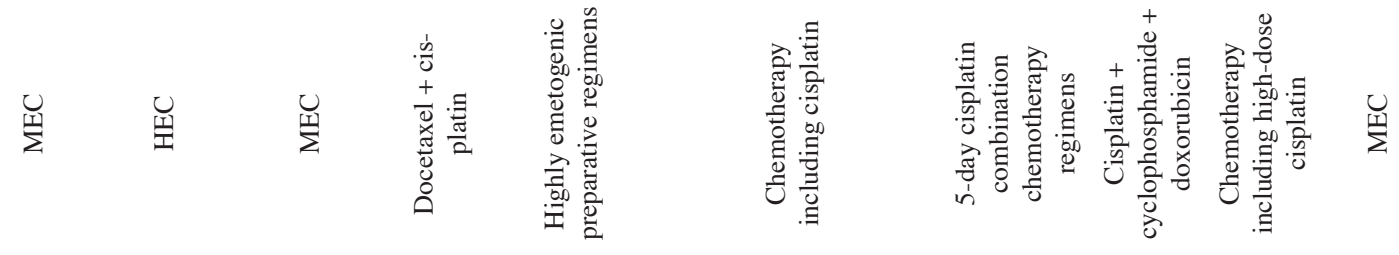

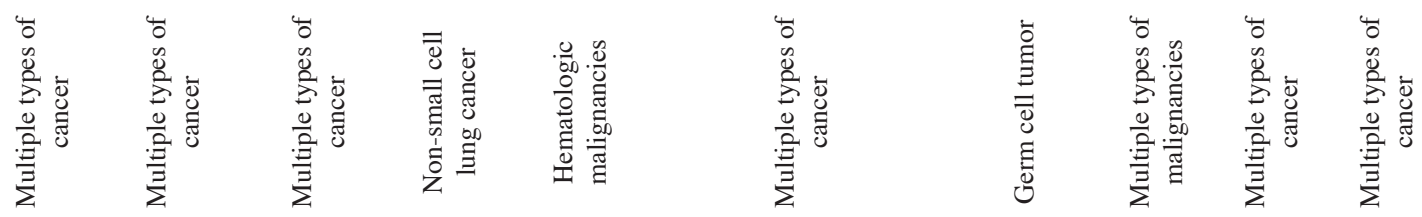

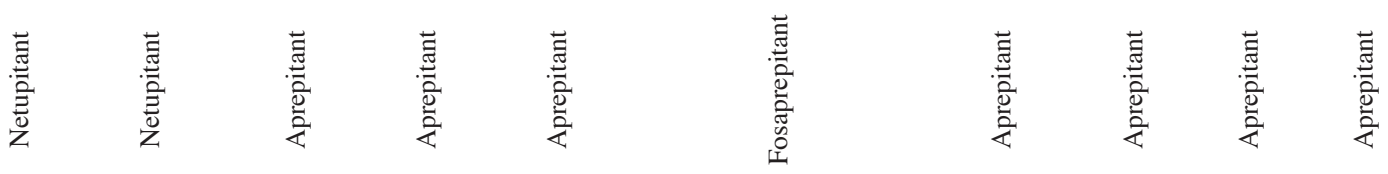

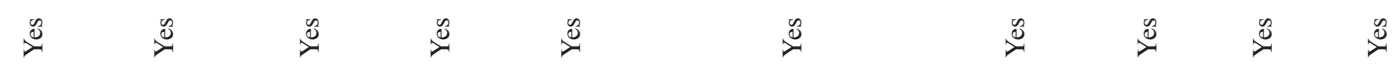

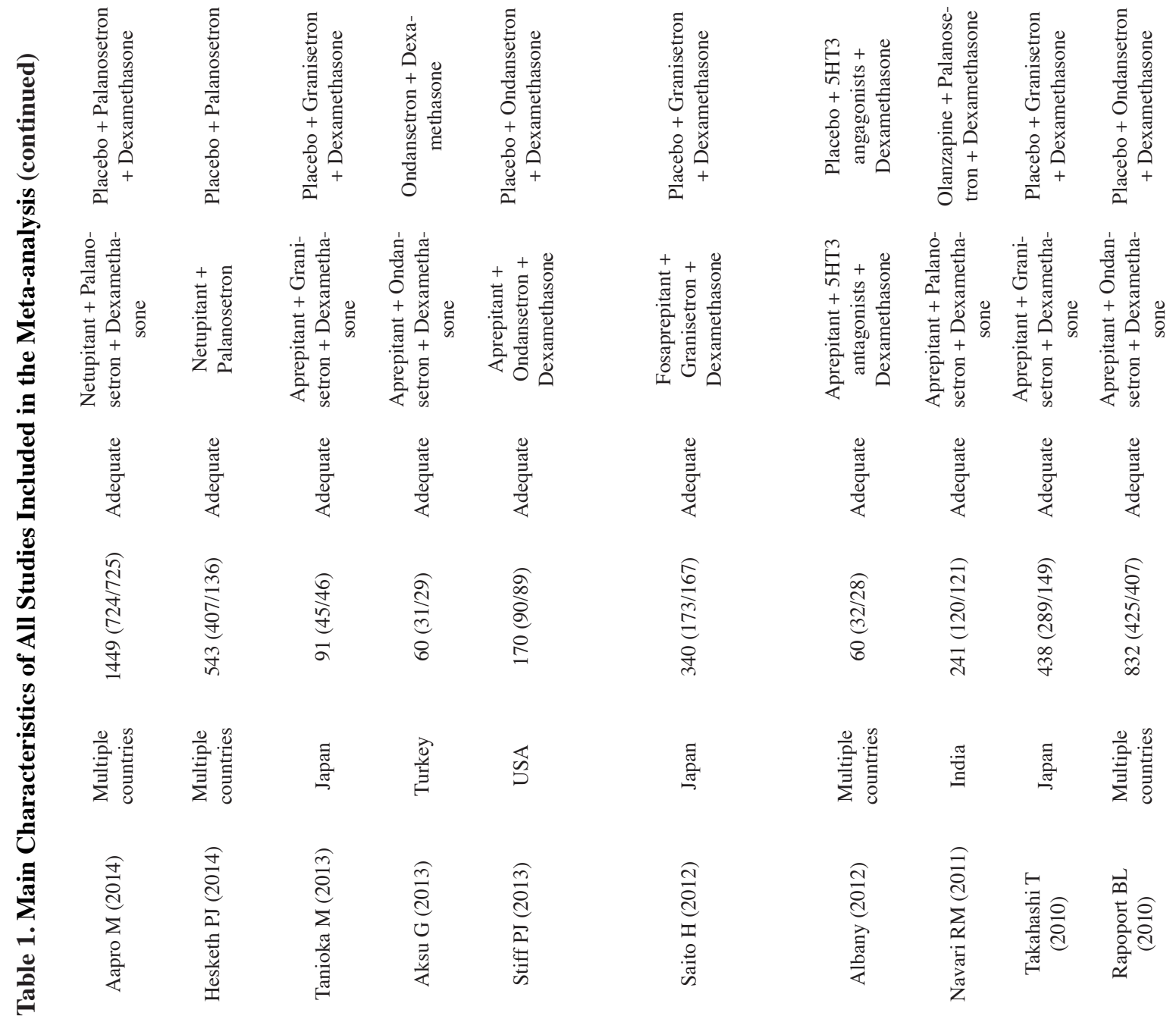


IIIUI:H:

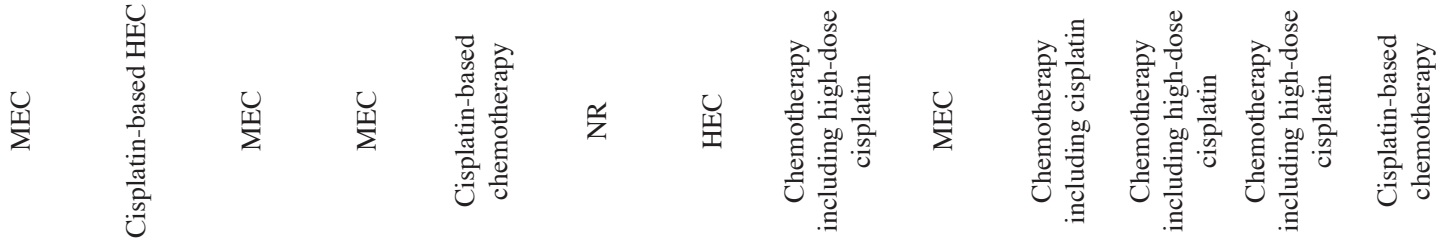

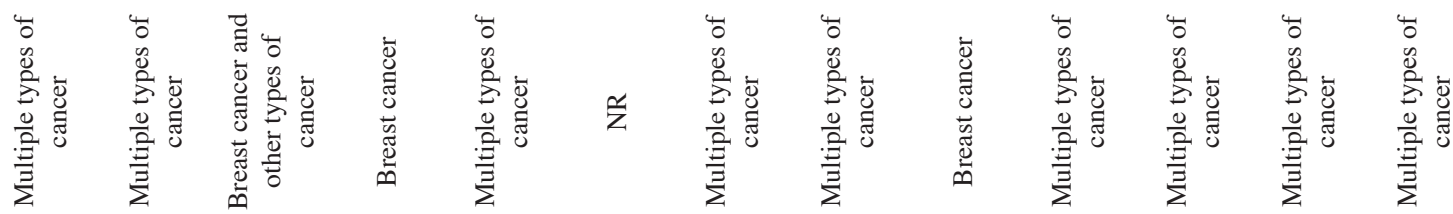

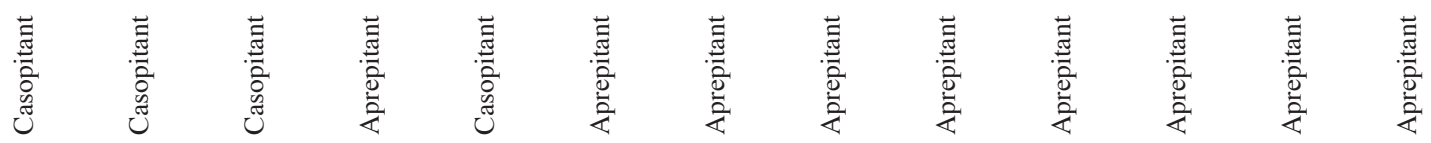

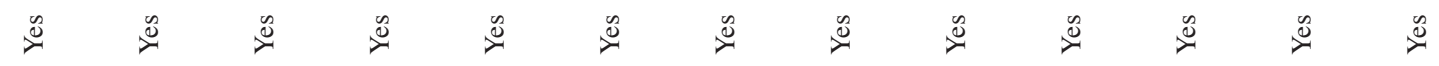

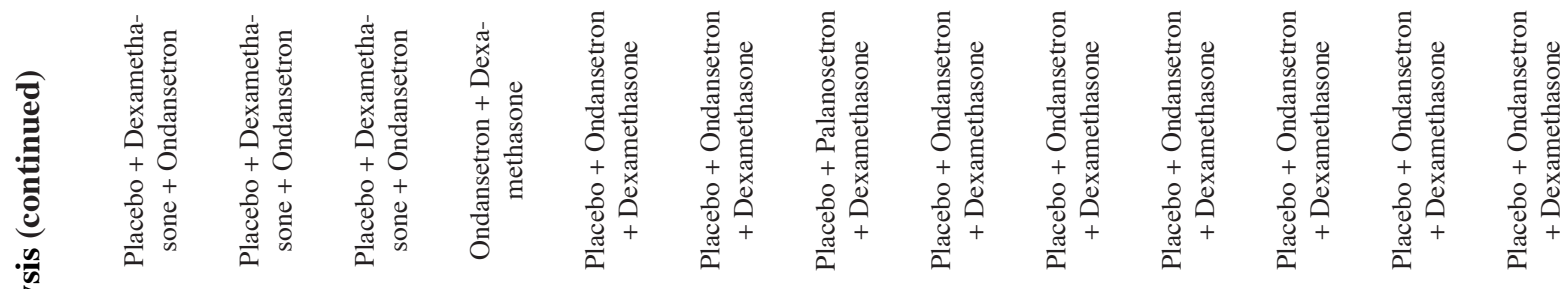

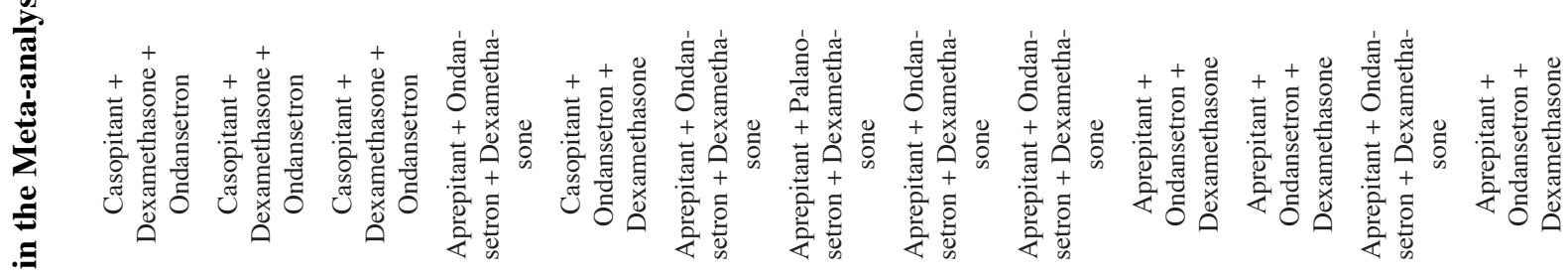

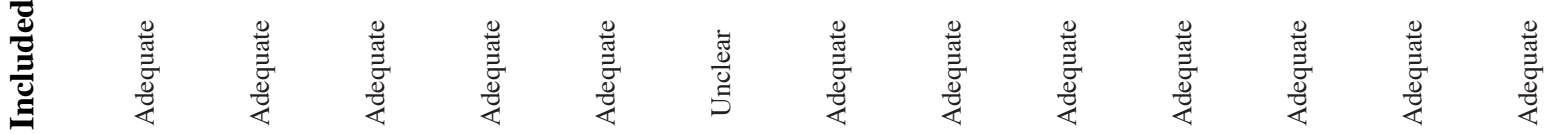

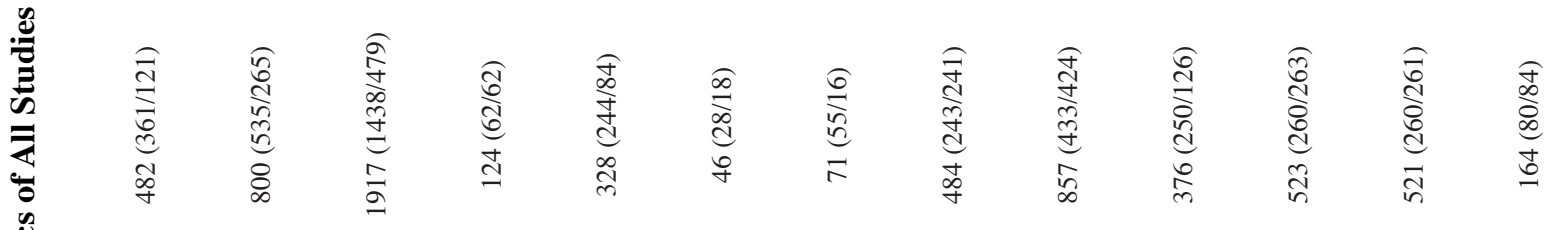

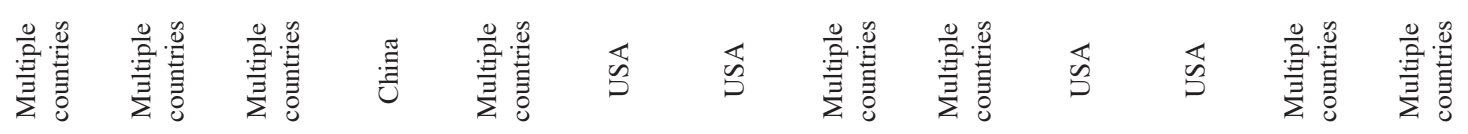

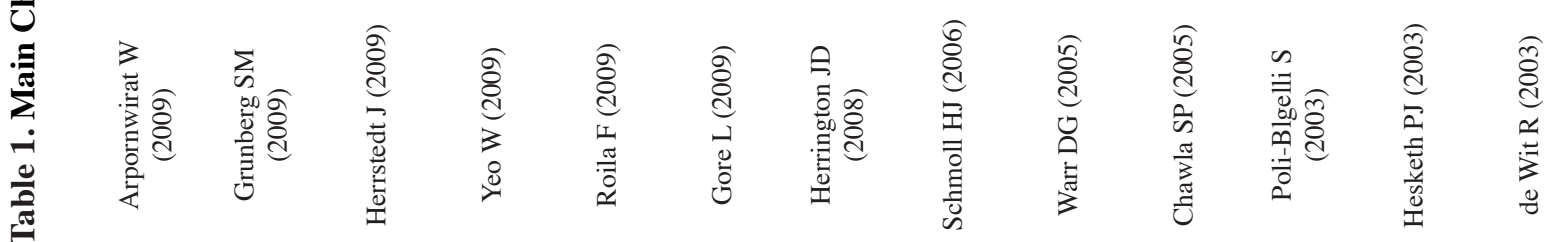

1666 Asian Pacific Journal of Cancer Prevention, Vol 17, 2016 

al., 2015; Nasu et al., 2015; Roila et al., 2015). All of the included studies were fully published, and all of them were reported in English. There were no major divergences in the definition of outcomes among the selected studies. The characteristics of included 38 studies were listed in Table 1. There were 16 studies carried out in multiple countries (Campos et al., 2001; Cocquyt et al., 2001; Van Belle et al., 2002; Hesketh et al., 2003; de Wit et al., 2004; Warr et al., 2005b; Schmoll et al., 2006; Arpornwirat et al., 2009; Grunberg et al., 2009; Herrstedt et al., 2009; Roila et al., 2009; Rapoport et al., 2010; Albany et al., 2012; Aapro et al., 2014; Hesketh et al., 2014; Kang et al., 2015), and 22 studies performed in single country (Hesketh et al., 1999; Navari et al., 1999; Chawla et al., 2003; Poli-Bigelli et al., 2003; Herrington et al., 2008; Gore et al., 2009; Yeo et al., 2009; Takahashi et al., 2010; Navari et al., 2011; Aksu et al., 2013; Saito et al., 2013; Stiff et al., 2013; Tanioka et al., 2013; Hu et al., 2014; Ito et al., 2014; Roila et al., 2014; Schmitt et al., 2014; Badar et al., 2015; Bakhshi et al., 2015; Kitayama et al., 2015; Nasu et al., 2015; Roila et al., 2015). Patients with multiple types of malignancies (Hesketh et al., 1999; Navari et al., 1999; Campos et al., 2001; Cocquyt et al., 2001; Van Belle et al., 2002; Chawla et al., 2003; Hesketh et al., 2003; Poli-Bigelli et al., 2003; de Wit et al., 2004; Schmoll et al., 2006; Herrington et al., 2008; Arpornwirat et al., 2009; Grunberg et al., 2009; Roila et al., 2009; Rapoport et al., 2010; Takahashi et al., 2010; Navari et al., 2011; Saito et al., 2013; Tanioka et al., 2013; Aapro et al., 2014; Hesketh et al., 2014; Hu et al., 2014; Bakhshi et al., 2015; Kang et al., 2015; Kitayama et al., 2015; Roila et al., 2015), Hematological malignancies (Albany et al., 2012; Stiff et al., 2013; Schmitt et al., 2014; Badar et al., 2015; Nasu et al., 2015), non-small cell lung cancer (NSCLC) (Aksu et al., 2013; Ito et al., 2014), breast cancer (Warr et al., 2005b; Herrstedt et al., 2009; Yeo et al., 2009; Roila et al., 2014) were all included in this research. In 30 out of included studies, NK1-RAs were used in combination with 5-HT3-RAs plus dexamethasone (Hesketh et al., 1999; Navari et al., 1999; Chawla et al., 2003; Hesketh et al., 2003; Poli-Bigelli et al., 2003; de Wit et al., 2004; Warr et al., 2005b; Schmoll et al., 2006; Herrington et al., 2008; Arpornwirat et al., 2009; Gore et al., 2009; Grunberg et al., 2009; Herrstedt et al., 2009; Roila et al., 2009; Yeo et al., 2009; Rapoport et al., 2010; Takahashi et al., 2010; Navari et al., 2011; Albany et al., 2012; Aksu et al., 2013; Saito et al., 2013; Stiff et al., 2013; Tanioka et al., 2013; Aapro et al., 2014; Hu et al., 2014; Ito et al., 2014; Roila et al., 2014; Schmitt et al., 2014; Bakhshi et al., 2015; Kitayama et al., 2015). For different types of NK1-RAs, most of the researches chose aprepitant (Navari et al., 1999; Campos et al., 2001; Cocquyt et al., 2001; Van Belle et al., 2002; Chawla et al., 2003; Hesketh et al., 2003; Poli-Bigelli et al., 2003; de Wit et al., 2004; Warr et al., 2005b; Schmoll et al., 2006; Herrington et al., 2008; Gore et al., 2009; Yeo et al., 2009; Rapoport et al., 2010; Takahashi et al., 2010; Navari et al., 2011; Albany et al., 2012; Aksu et al., 2013; Stiff et al., 2013; Tanioka et al., 2013; Hu et al., 2014; Ito et al., 2014; Roila et al., 2014; Schmitt et al., 2014; Badar et al., 2015; Bakhshi et al., 2015; Kang et al., 2015; Nasu et al., 2015; Roila et 


\section{Table 2. Subgroup Analyses of Complete Response}

Endpoints and subgroups
Acute CR
Types of NK1-RAs
Aprepitant
Casopitant
Fosaprepitant
Others

No. of included studies 34 26

4

2

2

Routine of administration

$$
\begin{aligned}
& \text { Orally } \\
& \text { Intravenously }
\end{aligned}
$$

Both

Age of included patients

Children or adolescents

Adults

3

Both

Drugs in control arm

$$
\text { Placebo }
$$

Others

Type of malignancies

$$
\text { Solid tumor }
$$

Hematologic malignancy

Both

Antiemetic drugs in combination with NK1-RAs

5-HT3-RAs
5-HT3-RAs+Dexamethasone
Others
Delayed CR

Types of NK1-RAs

$\begin{array}{ll}\text { Aprepitant } & 25 \\ \text { Casopitant } & \\ \text { Fosaprepitant } & 2 \\ \text { Others } & 2\end{array}$

Routine of administration

$\begin{array}{lc}\text { Orally } & 28 \\ \text { Intravenously } & 2 \\ \text { Both } & 3\end{array}$

Age of included patients

Children or adoles cents

Adults

Both

3

Drugs in control arm

$$
\text { Placebo }
$$

Others

Type of malignancies

$$
\text { Solid tumor }
$$

Hematologic malignancy

Both

Weight $(\%)$
100

73.79
14.86
3.45
7.9

85.01

3.45

11.54

7.93

90.4

1.67

77.86

22.14

85.24

4.89

9.88

8.66

80.23

11.12

100

69.38

17.31

4.45

8.86

82.4

4.45

13.15

5.21

93.67

1.11

79.45

20.55

87.82

4.13

8.05
OR (95\%CI)

1.547 (1.269-1.884)

$P$ value

0

1.480 (1.143-1.918)

0.003

1.530 (1.110-2.109)

0.009

3.504 (1.731-7.092)

1.499 (1.032-2.177)

0

0.034

1.598 (1.340-1.906)

0

3.504 (1.731-7.092)

0.774 (0.224-2.673)

0.685

2.863 (1.261-6.497)

1.438 (1.173-1.762)

0.012

0

0.01

1.838 (1.577-2.141)

0

0.682 (0.397-1.170)

0.165

1.466 (1.201-1.790)

0

3.427 (1.635-7.180)

0.001

$1.636(0.502-5.336)$

0.414

1.920 (1.319-2.793)

0.001

1.702 (1.414-2.050)

0

0.552 (0.191-1.601)

0.274

1.885 (1.671-2.126)

0

1.866 (1.588-2.193)

0

2.074 (1.764-2.439)

0

1.809 (1.157-2.828)

0.009

1.764 (1.099-2.831)

0.019

1.878 (1.628-2.167)

1.809 (1.157-2.828)

0

0.009

2.024 (1.699-2.412)

0

2.417 (1.049-5.569)

0

1.847 (1.636-2.087)

0.038

3.000 (1.046-8.603)

0.041

2.007 (1.775-2.268)

0

1.444 (1.084-1.923)

0.012

1.885 (1.661-2.139)

0

$1.880(1.199-2.947)$

0.006

$1.887(0.938-3.796)$

0.075 
Table 2. Subgroup Analyses of Complete Response (continued)

Antiemetic drugs in combination

with NK1-RAs

\begin{tabular}{|c|c|c|c|c|}
\hline 5-HT3-RAs & 3 & 7.07 & $2.711(1.913-3.842)$ & 0 \\
\hline 5-HT3-RAs+Dexamethasone & 26 & 84.25 & $1.847(1.627-2.098)$ & 0 \\
\hline Others & 4 & 8.68 & $1.744(0.988-3.079)$ & 0.055 \\
\hline Overall CR & 32 & 100 & $1.855(1.668-2.062)$ & 0 \\
\hline \multicolumn{5}{|l|}{ Types of NK1-RAs } \\
\hline Aprepitant & 24 & 66.21 & $1.817(1.584-2.086)$ & 0 \\
\hline Casopitant & 4 & 19.01 & $2.080(1.769-2.445)$ & 0 \\
\hline Fosaprepitant & 2 & 4.33 & $1.556(0.662-3.660)$ & 0.311 \\
\hline Others & 2 & 10.45 & $1.728(1.113-2.685)$ & 0.015 \\
\hline \multicolumn{5}{|l|}{ Routine of administration } \\
\hline Orally & 28 & 83.03 & $1.826(1.619-2.059)$ & 0 \\
\hline Intravenously & 2 & 4.33 & $1.556(0.662-3.660)$ & 0.311 \\
\hline Both & 2 & 12.64 & $2.096(1.635-2.688)$ & 0 \\
\hline \multicolumn{5}{|l|}{ Age of included patients } \\
\hline Children or adolescents & 3 & 3.98 & $2.807(1.765-4.465)$ & 0 \\
\hline Adults & 28 & 95.36 & $1.811(1.628-2.016)$ & 0 \\
\hline Both & 1 & 0.65 & $4.105(1.151-14.648)$ & 0.03 \\
\hline \multicolumn{5}{|l|}{ Drugs in control arm } \\
\hline Placebo & 24 & 86.64 & $1.927(1.765-2.103)$ & 0 \\
\hline Others & 8 & 13.36 & $1.203(0.891-1.623)$ & 0.227 \\
\hline \multicolumn{5}{|l|}{ Type of malignancies } \\
\hline Solid tumor & 24 & 86.29 & $1.840(1.657-2.043)$ & 0 \\
\hline Hematologic malignancy & 4 & 7.2 & $2.049(1.381-3.040)$ & 0 \\
\hline Both & 4 & 6.51 & $2.016(0.872-4.661)$ & 0.101 \\
\hline \multicolumn{5}{|l|}{$\begin{array}{l}\text { Antiemetic drugs in combination } \\
\text { with NK1-RAs }\end{array}$} \\
\hline 5-HT3-RAs & 4 & 8.13 & $2.286(1.543-3.388)$ & 0 \\
\hline 5-HT3-RAs+Dexamethasone & 28 & 91.87 & $1.819(1.632-2.029)$ & 0 \\
\hline Others & NA & NA & NA & NA \\
\hline
\end{tabular}

No.= umber; $\mathrm{OR}=$ odds ratio; $95 \% \mathrm{CI}=95 \%$ confidence interval; $\mathrm{CR}=$ complete response; NK1-RAs=neurokinin-1 receptor antagonists; 5-HT3RAs=5-hydroxytryptamine receptor antagonists; NA=not applicable. An OR more than 1 favored the NK1-RAs group, whereas an OR less than 1 favored the control group.

al., 2015), 2 studies used fosaprepitant (Saito et al., 2013; Kitayama et al., 2015), 4 researches focused on casopitant (Arpornwirat et al., 2009; Grunberg et al., 2009; Herrstedt et al., 2009; Roila et al., 2009), while the remaining 3 articles used other types of NK1-RAs (Hesketh et al., 1999; Aapro et al., 2014; Hesketh et al., 2014). There were three researches focusing on the efficacy of NK1-RAs in children or adolescents (Gore et al., 2009; Bakhshi et al., 2015; Kang et al., 2015).

\section{Complete Response}

32 RCTs were included in the analysis of CR in the overall phase. In the NK1-RAs groups, 5354 out of 7561 patients $(70.8 \%)$ had a CR, while only 2966 of 5259 patients $(56.0 \%)$ achieved CR in the control groups (OR: 1.855, 95\% CI: 1.668-2.062), $(P<0.001)$ (Figure 2A). Although no significant heterogeneity was observed in most of the comparisons, we sought to minimize the potential heterogeneity and probe into detailed results in the sub-population by performing a subgroup analysis. Among patients receiving aprepitant, the CR rate was also significantly higher in the treatment group $(64.2 \%$ vs $51.4 \%$, OR: $1.817,95 \% \mathrm{CI}: 1.584-2.086),(P<0.001)$. However, we found that patients treated with fosaprepitant intravenously did not increase the CR rate of CINV (OR: 1.556, 95\%CI: $0.662-3.660),(P=0.311)$.

During the acute phase, we had sufficient data from 34 RCTs and 13561 patients. The CR rate in acute phase of all included patients was $82.9 \%$. In the NK1-RAs group, $85.1 \%$ of patients (6866 out 8066) had CR. Meanwhile, only $79.6 \%$ of patients (4372 out 5495) experienced CR in the patients did not receive NK1-RAs (OR: $1.547,95 \% \mathrm{CI}$ : 1.269-1.884, $P<0.001$ ) (Figure 2B). In the subgroup analysis, we demonstrated that patients treated with NK1RAs orally (OR: $1.598,95 \% \mathrm{CI}: 1.340-1.906, P<0.001)$ or intravenously (OR: 3.504, 95\%CI: 1.731-7.092, $P<0.001$ ) 
Dong-Mei Yuan et al

Table 3. Reported Adverse Events of Included Researches

\begin{tabular}{|c|c|c|c|c|}
\hline Adverse events & No. of patients & No. at risk (\%) & OR $(95 \% \mathrm{CI})$ & $P$ value \\
\hline Any adverse events & 10694 & $7216(67.5)$ & & \\
\hline NK1-RAs group & 6447 & 4445 (68.9) & $1.061(0.968-1.162)$ & 0.204 \\
\hline Control group & 4247 & $2771(65.2)$ & & \\
\hline Anemia & 2202 & $393(17.8)$ & & \\
\hline NK1-RAs group & 1371 & $230(16.8)$ & $0.932(0.740-1.173)$ & 0.547 \\
\hline Control group & 831 & $163(19.6)$ & & \\
\hline Anorexia & 7791 & $1308(16.8)$ & & \\
\hline NK1-RAs group & 4576 & $763(16.7)$ & $1.053(0.919-1.206)$ & 0.458 \\
\hline Control group & 3217 & $545(16.9)$ & & \\
\hline Asthenia/Fatigue & 10326 & 1924 (18.6) & & \\
\hline NK1-RAs group & 6186 & 1170 (18.9) & $1.103(0.992-1.227)$ & 0.071 \\
\hline Control group & 4140 & $754(18.2)$ & & \\
\hline Constipation & 13654 & $2000(14.6)$ & & \\
\hline NK1-RAs group & 7980 & 1107 (13.9) & $0.836(0.755-0.924)$ & 0 \\
\hline Control group & 5674 & $893(15.7)$ & & \\
\hline Diarrhea & 7117 & 907 (12.7) & & \\
\hline NK1-RAs group & 4008 & $566(14.1)$ & $1.241(1.063-1.447)$ & 0.006 \\
\hline Control group & 3109 & $341(11.0)$ & & \\
\hline Dizziness & 1599 & $166(10.4)$ & & \\
\hline NK1-RAs group & 1047 & $124(13.4)$ & $1.385(0.948-2.022)$ & 0.092 \\
\hline Control group & 552 & $42(7.6)$ & & \\
\hline Epigastric/Abdominal pain & 1740 & $195(11.2)$ & & 0.164 \\
\hline NK1-RAs group & 1009 & $108(10.7)$ & $0.804(0.591-1.093)$ & \\
\hline Control group & 731 & $87(11.9)$ & & \\
\hline Headache & 9048 & $1104(12.2)$ & & \\
\hline NK1-RAs group & 5395 & $637(11.8)$ & $0.905(0.790-1.037)$ & 0.152 \\
\hline Control group & 3653 & $467(12.8)$ & & \\
\hline Heartburn & 1138 & $57(5.0)$ & & \\
\hline NK1-RAs group & 577 & $26(4.5)$ & $0.818(0.480-1.394)$ & 0.46 \\
\hline Control group & 561 & $31(5.5)$ & & \\
\hline Hiccups & 5229 & $737(14.1)$ & & \\
\hline NK1-RAs group & 3144 & $474(17.8)$ & $1.265(1.064-1.505)$ & 0.008 \\
\hline Control group & 2085 & $263(12.6)$ & & \\
\hline Insomnia & 2002 & $78(3.9)$ & & \\
\hline NK1-RAs group & 1080 & $32(3.0)$ & $0.493(0.308-0.789)$ & 0.003 \\
\hline Control group & 922 & $46(5.0)$ & & \\
\hline Leukopenia & 3805 & $932(24.5)$ & & \\
\hline NK1-RAs group & 2606 & $584(22.4)$ & $0.996(0.839-1.183)$ & 0.965 \\
\hline Control group & 1199 & $348(29.0)$ & & \\
\hline Neutropenia & 8433 & $1642(19.5)$ & & \\
\hline NK1-RAs group & 5333 & $1100(20.6)$ & $1.017(0.894-1.158)$ & 0.795 \\
\hline Control group & 3100 & $542(17.5)$ & & \\
\hline Pruritus & 1014 & $8(0.8)$ & & \\
\hline NK1-RAs group & 515 & $5(1.0)$ & $1.533(0.397-5.925)$ & 0.536 \\
\hline Control group & 499 & $3(0.6)$ & & \\
\hline
\end{tabular}


Table 3. Reported Adverse Events of Included Researches (continued)

$\begin{array}{ccccc}\text { Tarchycardia } & 1379 & 19(13.8) & & 0.637(0.256-1.587) \\ \text { NK1-RAs group } & 833 & 8(9.6) & 0.333 \\ \text { Control group } & 546 & 11(2.0) & & \\ \text { Thrombocytopenia } & 1582 & 300(19.0) & & 0.74 \\ \text { NK1-RAs group } & 930 & 165(21.6) & 0.956(0.730-1.250) & \\ \text { Control group } & 652 & 135(20.7) & & \end{array}$

No.= umber; OR=odds ratio; 95\%CI=95\% confidence interval; NK1-RAs=neurokinin-1 receptor antagonists. An OR less than 1 favored the NK1RAs group, whereas an OR more than 1 favored the control group.
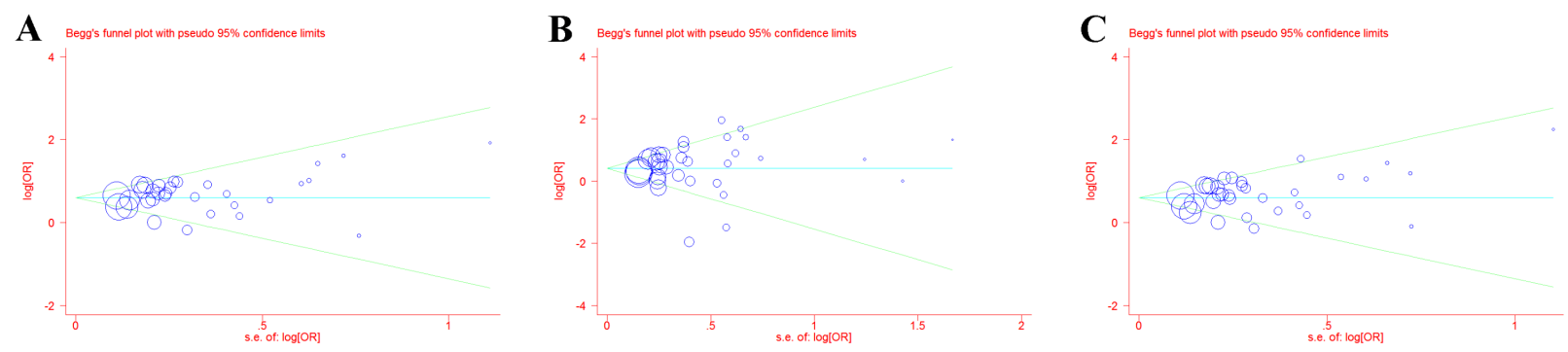

Figure 3. Funnel Plots of Odds Ratios for Included Studies for (A) Overall Complete Response (CR), (B) CR in the Acute Phase, and (C) CR in the Delayed Phase

both could increase the CR rate in acute phase. However, when patients received NK1-RAs intravenously in the first day and followed by oral NK1-RAs, the CR rate was not improved (OR: 0.774, 95\%CI: 0.224-2.673, $P=0.685$ ). Furthermore, NK1-RAs were often used in combination with other antiemetic drugs, they had higher rate of CR in acute phase when used together with 5-HT3-RAs (OR: $1.920,95 \% \mathrm{CI}: 1.319-2.793, P=0.001)$ or 5 -HT3RAs+Dexamethasone (OR: 1.702, 95\%CI: 1.414-2.050, $P<0.001)$. Nonetheless, when NK1-RAs were given with other antiemetic regimens (such as dexamethasone only), the CR rate in acute phase was not increased (OR: 0.552 , 95\%CI: 0.191-1.601, $P=0.274)$.

Data from 33 RCTs and 13385 patients were included in the analysis for the evaluation of CR rate in the delayed phase. There was again a significantly greater frequency of CR among patients given with NK1-RAs $(71.4 \%$ vs 58.2\%, OR: $1.885,95 \%$ CI: $1.671-2.126, P<0.001$ ) (Figure $2 \mathrm{C})$. And details about the results of subgroup analyses were shown in Table 2.

We should emphasis that NK1-RAs had increased CR rate in children or adolescents during overall phase (OR: 2.807 95\%CI: $1.765-4.465, P<0.001$ ), acute phase (OR: 2.863 95\%CI: 1.261-6.497, $P=0.012$ ), and delayed phase (OR: $2.417,95 \%$ CI: $1.049-5.569, P<0.001)$. It meant that the antiemetic efficacy was also improved by the addition of NK1-RAs in children.

\section{Other efficacy outcomes}

Other efficacy outcomes including the rate of appearance of nausea, vomiting and need of rescue therapy in the overall phase. For all these outcomes, patients receiving NK1-RAs showed significant advantages compared to patients in control group: (i) incidence of nausea (29 RCTs and 12554 patients included): $45.2 \%$ vs $45.9 \%$, OR: $0.834,95 \% \mathrm{CI}: 0.771-0.901, P<0.001$; (ii) occurrence of vomiting (31 RCTs and 13075 patients analyzed): $22.6 \%$ vs $38.9 \%$, OR: $0.451,95 \%$ CI: $0.416-$ $0.490, P<0.001$; (iii) use of rescue drugs (18 RCTs with 5102 patients were calculated): $23.5 \%$ vs $34.1 \%$, OR: 0.660, 95\%CI: $0.578-0.751, P<0.001$.

\section{Adverse events}

35 out of 38 included studies reported the safety of NK1-RAs. Toxicity was described according to the National Cancer Institute (NCI) Common Terminology Criteria for Adverse Events (CTCAE) in most studies. The adverse events reported in more than three researches were included in the final analysis of toxicity. The mostly reported adverse events were constipation, headache, and neutropenia. We demonstrated that the addition of NK1RAs did not increase the incidence of patients experienced no less than one adverse event $(68.9 \%$ vs $65.2 \%$, OR: 1.061,95\%CI: $0.968-1.162, P=0.204)$. However, we found that constipation $(13.9 \%$ vs $15.7 \%$, OR: $0.836,95 \% \mathrm{CI}$ : $0.755-0.924, P<0.001)$ and insomnia (3.0\% vs 5.0\%, OR: 0.493 ,95\%CI: $0.308-0.789, P=0.003$ ) were more common in the patients of control groups, whereas diarrhea $(14.1 \%$ vs $11.0 \%$, OR: $1.241,95 \%$ CI: $1.063-1.447, P=0.006)$ and hiccups $(17.8 \%$ vs $12.6 \%$, OR: $1.265,95 \% \mathrm{CI}: 1.064-$ $1.505, P=0.008)$ were more frequently to be detected in the patients receiving NK1-RAs. And the incidence rate of other adverse events, such as anemia, anorexia, asthenia, dizziness, etc, was not significantly different in two groups. Details about the analyses of toxicity were reported in Table 3.

Among the three researches included children or teenagers, Kang HJ, et al (Kang et al., 2015) and Gore L, et al (Gore et al., 2009) evaluated the incidence of any adverse events. The pooled analysis showed that the difference between NK1-RAs groups and control groups was not significant $(79.9 \%$ vs $76.8 \%$, OR: $1.193,95 \% \mathrm{CI}$ : 
$0.717-1.983, P=0.497)$. It demonstrated that NK1-RAs were well tolerable in children and teenagers.

\section{Bias analysis}

Begg's funnel plot and Egger's test were performed to assess the publication bias of the included trials. The shapes of the funnel plot for the data of CR in acute, delayed and overall phase did not reveal any evidence of obvious asymmetry (Figure 3). Furthermore, Egger's test was used to provide statistical difference (acute CR: $P=0.514$, delayed CR: $P=0.745$, overall CR: $P=0.593$ ).

\section{Discussion}

More and more effective and better tolerated agents have been developed to prevent CINV. With the proper use of antiemetic drugs, CINV can be prevented in almost $70 \%$ to $80 \%$ of patients receiving chemotherapy. Till now, 5-HT3-RAs, NK1-RAs, and corticosteroids are considered to be the most effective therapeutic combination. In present study, we performed a systematic review and metaanalysis, and tried to figure out the efficacy and tolerability of the addition of NK1-RAs in the prevention of CINV. At the beginning, we demonstrated that patients receiving NK1-RAs had significantly higher CR rate compared to patients without NK1-RAs during the overall, acute, and delayed phase. These results provided evidence that the addition of NK1-RAs brought benefits for patients receiving chemotherapy. According to the positive results about NK1-RAs in the previous clinical researches, NK1-RAs have been added in several guidelines of the treatment of CINV (Herrstedt and Roila, 2009; Basch et al., 2011; Jordan et al., 2014). In the above guidelines, patients receiving HEC or MEC were recommended to be given with the combination of 5-HT3-RAs, NK1RAs, and dexamethasone during the acute phase. And our research further confirmed the addition of NK1-RAs to other antiemetic regimens in the prevention of CINV.

As the first NK1-RAs approved by the FDA, aprepitant was the mostly often tested agent in our identified trials. And patients given aprepitant showed improvement in all of the interested outcomes, including CR rate, incidence of nausea and vomiting, and need of rescue therapy. However, in the subgroup analysis, we found that although patients treated with fosaprepitant intravenously had the trend to increase the CR rate of CINV, the difference between two groups was not significant. Fosaprepitant is an intravenous formulation of aprepitant that could convert to aprepitant in 30 minutes (Navari, 2007), and an intravenous dose of $115 \mathrm{mg}$ is area under the curve bioequivalent to aprepitant $125 \mathrm{mg}$ orally (Lasseter et al., 2007). Why the efficacy of fosaprepitant was not similar to aprepitant? One explanation to this question is that there were only two researches (Saito et al., 2013; Kitayama et al., 2015) and 375 patients included for the analysis of fosaprepitant, while Kitayama $\mathrm{H}$, et al (Kitayama et al., 2015) got negative results. Secondly, studies used the combination of intravenous fosaprepitant and oral aprepitant were not included in the analysis of fosparepitant. Whether single intravenous fosaprepitant or combination of fosaprepitant and aprepitant could be an ideal choice for NK1-RAs? We need further clinical trials to solve this problem.

Currently, control of nausea is more difficult than control of vomiting (Grunberg et al., 2004). Previous results of NK1-RAs on the control of nausea were inconsistent. Saito H et al (Saito et al., 2013) did not find significant differences in terms of control of nausea in the overall, acute, and delayed phases. However, a combined analysis of the Poli-Bligelli et al trial (Poli-Bigelli et al., 2003) and Hesketh et al trial (Hesketh et al., 2003) showed a significant decrease of the incidence of nausea (Warr et al., 2005a). Our research got an agreement to this point, and demonstrated that the incidence of nausea and vomiting both significantly decreased after addition of NK1-RAs.

Another conclusion that could be drawn from our study was that NK1-RAs were well tolerated. The most frequently reported adverse events were constipation, headache, and neutropenia. It is quite interesting to find that patients receiving NK1-RAs had more risks of diarrhea and hiccups, while the risk of constipation and insomnia was decreased significantly. Diarrhea is a relatively common adverse effect from cytotoxic antineoplasic treatment and may be debilitating and potentially life threatening and dose limiting (Wadler et al., 1998). Men had a significantly higher incidence of hiccups post-chemotherapy, while women had significantly higher rates of vomiting and nausea (Liaw et al., 2001). We should take these results in consideration before we choose NK1-RAs for the patients receiving chemotherapy. Furthermore, the efficacy of safety of aprepitant have not been fully tested in other disease in which the substance $P /$ NK-1 receptor system is involved (such as cancer, alcoholism, etc), clinical trials are now in progress (Munoz and Covenas, 2013).

A strong point of our study is that we pooled the results of NK1-RAs in children and teenagers. There are more and more researches focusing on the application of NK1RAs in children. In a retrospective study, aprepitant was given to patients as young as 11 months old (Shillingburg and Biondo, 2014). There were three RCTs evaluated the efficacy and tolerability of NK1-RAs in children and adolescents in the identified studies. The pooled analysis demonstrated NK1-RAs were effective and safe in children and teens receiving chemotherapy, and different types of NK1-RAs might be another choice to prevent CINV in such patients.

Our study of CR rate in acute phase should be concerned with the problem of heterogeneity. There was significant heterogeneity among the 34 valuable studies used to assess the effect of NK1-RAs during acute phase $\left(\mathrm{I}^{2}=68.2 \%, P<0.001\right)$. Some diversity in the designs of the different studies contributes to the heterogeneity. For example, there were different types of chemotherapy. The choices for therapy greatly influence the incidence of nausea and vomiting. To be continued, the present study also has the typical limitations of the meta-analytical methodology. Our findings and interpretations were limited by the quality and quantity of available evidence on the effects of NK1-RAs on the prevention of CINV, and only published data were used in this study. Publication 
bias is another concern in all forms of meta-analysis.

Still, there are several questions remaining to be solved about the application of NK1-RAs. Firstly, what types of antiemetic drugs should be used together with NK1-RAs? In our research, the antiemetic efficacy seemed to be better when NK1-RAs were used along with 5-HT3-RAs or 5-HT3-RAs plus dexamethasone. To be followed, what is the optimal dose of NK1-RAs? We did not compared different dosage of NK1-RAs in this study. Arpornwirat W et al (Arpornwirat et al., 2009) identified casopitant 150mg as the minimally effective dose. And Roila F et al (Roila et al., 2009) did not found significant difference between different doses of NK1-RAs. These questions have not yet been solved, the role of NK1-RAs for patients receiving antineoplasic therapy is still under active investigation. We look forward to more clinical trials to solve the above two points.

In conclusion, despite some limitations, our metaanalysis suggests that the addition of NK1-RAs could increase the CR rate for the prevention of CINV during acute, delayed, and overall phase. Meanwhile, NK1-RAs could decrease the incidence of nausea, vomiting, and need of rescue therapy. The use of NK1-RAs might be associated with an increased risk of diarrhea and hiccups, and a decreased risk of constipation and insomnia. For children and adolescents patients, NK1-RAs were still effective and well tolerated.

\section{Acknowledgements}

This work was supported by the National Science Foundation of Jiangsu Province of China (No. BK20140736 to Dong-mei Yuan), the Standard Diagnosis and Treatment Program of Key Disease in Jiangsu Province (No. BL2013026 to Yong Song), and the National Natural Science Foundation of China (No. 81370172 to Yong Song).

\section{References}

Aapro M, Carides A, Rapoport BL, et al (2015). Aprepitant and fosaprepitant: a 10-year review of efficacy and safety. Oncologist, 20, 450-8.

Aapro M, Rugo H, Rossi G, et al (2014). A randomized phase III study evaluating the efficacy and safety of NEPA, a fixed-dose combination of netupitant and palonosetron, for prevention of chemotherapy-induced nausea and vomiting following moderately emetogenic chemotherapy. Ann Oncol, 25, 1328-33.

Aapro MS, Molassiotis A, Olver I (2005). Anticipatory nausea and vomiting. Support Care Cancer, 13, 117-21.

Aksu G, Dolasik I, Ensaroglu F, et al (2013). Evaluation of the efficacy of aprepitant on the prevention of chemotherapyinduced nausea and vomiting and quality of life with functional living index emesis. Balkan Med J, 30, 64-7.

Albany C, Brames MJ, Fausel C, et al (2012). Randomized, double-blind, placebo-controlled, phase III cross-over study evaluating the oral neurokinin-1 antagonist aprepitant in combination with a 5HT3 receptor antagonist and dexamethasone in patients with germ cell tumors receiving 5-day cisplatin combination chemotherapy regimens: a hoosier oncology group study. J Clin Oncol, 30, 3998-4003.
Altman DG, Bland JM (1997). Statistics notes. Units of analysis. $B m j, 314,1874$.

Arpornwirat W, Albert I, Hansen VL, et al (2009). Phase 2 trial results with the novel neurokinin-1 receptor antagonist casopitant in combination with ondansetron and dexamethasone for the prevention of chemotherapy-induced nausea and vomiting in cancer patients receiving moderately emetogenic chemotherapy. Cancer, 115, 5807-16.

Badar T, Cortes J, Borthakur G, et al (2015). Phase II, open label, randomized comparative trial of ondansetron alone versus the combination of ondansetron and aprepitant for the prevention of nausea and vomiting in patients with hematologic malignancies receiving regimens containing high-dose cytarabine. Biomed Res Int, 2015, 497597.

Bakhshi S, Batra A, Biswas B, et al (2015). Aprepitant as an add-on therapy in children receiving highly emetogenic chemotherapy: a randomized, double-blind, placebocontrolled trial. Support Care Cancer, 23, 3229-37.

Basch E, Prestrud AA, Hesketh PJ, et al (2011). Antiemetics: American Society of Clinical Oncology clinical practice guideline update. J Clin Oncol, 29, 4189-98.

Bloechl-Daum B, Deuson RR, Mavros $P$, et al (2006). Delayed nausea and vomiting continue to reduce patients' quality of life after highly and moderately emetogenic chemotherapy despite antiemetic treatment. J Clin Oncol, 24, 4472-8.

Campos D, Pereira JR, Reinhardt RR, et al (2001). Prevention of cisplatin-induced emesis by the oral neurokinin-1 antagonist, MK-869, in combination with granisetron and dexamethasone or with dexamethasone alone.J Clin Oncol, 19, 1759-67.

Chawla SP, Grunberg SM, Gralla RJ, et al (2003). Establishing the dose of the oral NK1 antagonist aprepitant for the prevention of chemotherapy-induced nausea and vomiting. Cancer, 97, 2290-300.

Cocquyt V, Van Belle S, Reinhardt RR, et al (2001). Comparison of L-758,298, a prodrug for the selective neurokinin-1 antagonist, L-754,030, with ondansetron for the prevention of cisplatin-induced emesis. Eur J Cancer, 37, 835-42.

de Wit R, Herrstedt J, Rapoport B, et al (2004). The oral NK(1) antagonist, aprepitant, given with standard antiemetics provides protection against nausea and vomiting over multiple cycles of cisplatin-based chemotherapy: a combined analysis of two randomised, placebo-controlled phase III clinical trials. Eur J Cancer, 40, 403-10.

DerSimonian R, Laird N (1986). Meta-analysis in clinical trials. Control Clin Trials, 7, 177-88.

Di Maio M, Bria E, Banna GL, et al (2013). Prevention of chemotherapy-induced nausea and vomiting and the role of neurokinin 1 inhibitors: from guidelines to clinical practice in solid tumors. Anticancer Drugs, 24, 99-111.

dos Santos LV, Souza FH, Brunetto AT, et al (2012). Neurokinin-1 receptor antagonists for chemotherapy-induced nausea and vomiting: a systematic review. J Natl Cancer Inst, 104, 1280-92.

Duval S, Tweedie R (2000). Trim and fill: A simple funnel-plotbased method of testing and adjusting for publication bias in meta-analysis. Biometrics, 56, 455-63.

Gore L, Chawla S, Petrilli A, et al (2009). Aprepitant in adolescent patients for prevention of chemotherapy-induced nausea and vomiting: a randomized, double-blind, placebocontrolled study of efficacy and tolerability. Pediatr Blood Cancer, 52, 242-7.

Grunberg SM (2007). Antiemetic activity of corticosteroids in patients receiving cancer chemotherapy: dosing, efficacy, and tolerability analysis. Ann Oncol, 18, 233-40.

Grunberg SM, Deuson RR, Mavros $P$, et al (2004). Incidence of chemotherapy-induced nausea and emesis after modern 
antiemetics. Cancer, 100, 2261-8.

Grunberg SM, Rolski J, Strausz J, et al (2009). Efficacy and safety of casopitant mesylate, a neurokinin 1 (NK1)-receptor antagonist, in prevention of chemotherapy-induced nausea and vomiting in patients receiving cisplatin-based highly emetogenic chemotherapy: a randomised, double-blind, placebo-controlled trial. Lancet Oncol, 10, 549-58.

Herrington JD, Jaskiewicz AD, Song J (2008). Randomized, placebo-controlled, pilot study evaluating aprepitant single dose plus palonosetron and dexamethasone for the prevention of acute and delayed chemotherapy-induced nausea and vomiting. Cancer, 112, 2080-7.

Herrstedt J, Apornwirat W, Shaharyar A, et al (2009). Phase III trial of casopitant, a novel neurokinin-1 receptor antagonist, for the prevention of nausea and vomiting in patients receiving moderately emetogenic chemotherapy. J Clin Oncol, 27, 5363-9.

Herrstedt J, Roila F (2009). Chemotherapy-induced nausea and vomiting: ESMO clinical recommendations for prophylaxis. Ann Oncol, 4, 156-8.

Hesketh PJ (2008). Chemotherapy-induced nausea and vomiting. $N$ Engl J Med, 358, 2482-94.

Hesketh PJ, Gralla RJ, Webb RT, et al (1999). Randomized phase II study of the neurokinin 1 receptor antagonist CJ-11,974 in the control of cisplatin-induced emesis. J Clin Oncol, 17, 338-43.

Hesketh PJ, Grunberg SM, Gralla RJ, et al (2003). The oral neurokinin-1 antagonist aprepitant for the prevention of chemotherapy-induced nausea and vomiting: a multinational, randomized, double-blind, placebo-controlled trial in patients receiving high-dose cisplatin--the Aprepitant Protocol 052 Study Group. J Clin Oncol, 21, 4112-9.

Hesketh PJ, Rossi G, Rizzi G, et al (2014). Efficacy and safety of NEPA, an oral combination of netupitant and palonosetron, for prevention of chemotherapy-induced nausea and vomiting following highly emetogenic chemotherapy: a randomized dose-ranging pivotal study. Ann Oncol, 25, 1340-6.

Higgins JP, Thompson SG (2002). Quantifying heterogeneity in a meta-analysis. Stat Med, 21, 1539-58.

$\mathrm{Hu}$ Z, Cheng Y, Zhang H, et al (2014). Aprepitant triple therapy for the prevention of chemotherapy-induced nausea and vomiting following high-dose cisplatin in Chinese patients: a randomized, double-blind, placebo-controlled phase III trial. Support Care Cancer, 22, 979-87.

Ito Y, Karayama M, Inui N, et al (2014). Aprepitant in patients with advanced non-small-cell lung cancer receiving carboplatin-based chemotherapy. Lung Cancer, 84, 259-64.

Jordan K, Gralla R, Jahn F, et al (2014). International antiemetic guidelines on chemotherapy induced nausea and vomiting (CINV): content and implementation in daily routine practice. Eur J Pharmacol, 722, 197-202.

Kang HJ, Loftus S, Taylor A, et al (2015). Aprepitant for the prevention of chemotherapy-induced nausea and vomiting in children: a randomised, double-blind, phase 3 trial. Lancet Oncol, 16, 385-94.

Kitayama H, Tsuji Y, Sugiyama J, et al (2015). Efficacy of palonosetron and 1-day dexamethasone in moderately emetogenic chemotherapy compared with fosaprepitant, granisetron, and dexamethasone: a prospective randomized crossover study. Int J Clin Oncol, 20, 1051-6.

Lasseter KC, Gambale J, Jin B, et al (2007). Tolerability of fosaprepitant and bioequivalency to aprepitant in healthy subjects. J Clin Pharmacol, 47, 834-40.

Liaw CC, Wang CH, Chang HK, et al (2001). Gender discrepancy observed between chemotherapy-induced emesis and hiccups. Support Care Cancer, 9, 435-41.
Martin AR, Pearson JD, Cai B, et al (2003a). Assessing the impact of chemotherapy-induced nausea and vomiting on patients' daily lives: a modified version of the Functional Living Index-Emesis (FLIE) with 5-day recall. Support Care Cancer, 11, 522-7.

Martin CG, Rubenstein EB, Elting LS, et al (2003b). Measuring chemotherapy-induced nausea and emesis. Cancer, $\mathbf{9 8 ,}$ 645-55.

Munoz M, Covenas R (2013). Safety of neurokinin-1 receptor antagonists. Expert Opin Drug Saf, 12, 673-85.

Nasu R, Nannya Y, Kurokawa M (2015). A randomized controlled study evaluating the efficacy of aprepitant for highly/moderately emetogenic chemotherapies in hematological malignancies. Int J Hematol, 101, 376-85.

Navari RM (2007). Fosaprepitant (MK-0517): a neurokinin-1 receptor antagonist for the prevention of chemotherapyinduced nausea and vomiting. Expert Opin Investig Drugs, 16, 1977-85.

Navari RM, Gray SE, Kerr AC (2011). Olanzapine versus aprepitant for the prevention of chemotherapy-induced nausea and vomiting: a randomized phase III trial. J Support Oncol, 9, 188-95.

Navari RM, Reinhardt RR, Gralla RJ, et al (1999). Reduction of cisplatin-induced emesis by a selective neurokinin-1receptor antagonist. L-754,030 Antiemetic Trials Group. $N$ Engl J Med, 340, 190-5.

Poli-Bigelli S, Rodrigues-Pereira J, Carides AD, et al (2003). Addition of the neurokinin 1 receptor antagonist aprepitant to standard antiemetic therapy improves control of chemotherapy-induced nausea and vomiting. Results from a randomized, double-blind, placebo-controlled trial in Latin America. Cancer, 97, 3090-8.

Rapoport BL, Jordan K, Boice JA, et al (2010). Aprepitant for the prevention of chemotherapy-induced nausea and vomiting associated with a broad range of moderately emetogenic chemotherapies and tumor types: a randomized, double-blind study. Support Care Cancer, 18, 423-31.

Roila F, Rolski J, Ramlau R, et al (2009). Randomized, double-blind, dose-ranging trial of the oral neurokinin-1 receptor antagonist casopitant mesylate for the prevention of cisplatin-induced nausea and vomiting. Ann Oncol, 20, 1867-73.

Roila F, Ruggeri B, Ballatori E, et al (2014). Aprepitant versus dexamethasone for preventing chemotherapy-induced delayed emesis in patients with breast cancer: a randomized double-blind study. J Clin Oncol, 32, 101-6.

Roila F, Ruggeri B, Ballatori E, et al (2015). Aprepitant versus metoclopramide, both combined with dexamethasone, for the prevention of cisplatin-induced delayed emesis: a randomized, double-blind study. Ann Oncol, 26, 1248-53.

Saito H, Yoshizawa H, Yoshimori K, et al (2013). Efficacy and safety of single-dose fosaprepitant in the prevention of chemotherapy-induced nausea and vomiting in patients receiving high-dose cisplatin: a multicentre, randomised, double-blind, placebo-controlled phase 3 trial. Ann Oncol, 24, 1067-73.

Saito M, Aogi K, Sekine I, et al (2009). Palonosetron plus dexamethasone versus granisetron plus dexamethasone for prevention of nausea and vomiting during chemotherapy: a double-blind, double-dummy, randomised, comparative phase III trial. Lancet Oncol, 10, 115-24.

Schmitt T, Goldschmidt H, Neben K, et al (2014). Aprepitant, granisetron, and dexamethasone for prevention of chemotherapy-induced nausea and vomiting after highdose melphalan in autologous transplantation for multiple myeloma: results of a randomized, placebo-controlled phase III trial. J Clin Oncol, 32, 3413-20. 
Schmoll HJ, Aapro MS, Poli-Bigelli S, et al (2006). Comparison of an aprepitant regimen with a multiple-day ondansetron regimen, both with dexamethasone, for antiemetic efficacy in high-dose cisplatin treatment. Ann Oncol, 17, 1000-6.

Shillingburg A, Biondo L (2014). Aprepitant and fosaprepitant use in children and adolescents at an academic medical center. J Pediatr Pharmacol Ther, 19, 127-31.

Stiff PJ, Fox-Geiman MP, Kiley K, et al (2013). Prevention of nausea and vomiting associated with stem cell transplant: results of a prospective, randomized trial of aprepitant used with highly emetogenic preparative regimens. Biol Blood Marrow Transplant, 19, 49-55.

Takahashi T, Hoshi E, Takagi M, et al (2010). Multicenter, phase II, placebo-controlled, double-blind, randomized study of aprepitant in Japanese patients receiving high-dose cisplatin. Cancer Sci, 101, 2455-61.

Tanioka M, Kitao A, Matsumoto K, et al (2013). A randomised, placebo-controlled, double-blind study of aprepitant in nondrinking women younger than 70 years receiving moderately emetogenic chemotherapy. Br J Cancer, 109, 859-65.

Tattersall FD, Rycroft W, Francis B, et al (1996). Tachykinin NK1 receptor antagonists act centrally to inhibit emesis induced by the chemotherapeutic agent cisplatin in ferrets. Neuropharmacol, 35, 1121-9.

Van Belle S, Lichinitser MR, Navari RM, et al (2002). Prevention of cisplatin-induced acute and delayed emesis by the selective neurokinin-1 antagonists, L-758,298 and MK-869. Cancer, 94, 3032-41.

Wadler S, Benson AB, 3rd, Engelking C, et al (1998). Recommended guidelines for the treatment of chemotherapyinduced diarrhea. J Clin Oncol, 16, 3169-78.

Warr DG, Grunberg SM, Gralla RJ, et al (2005a). The oral NK(1) antagonist aprepitant for the prevention of acute and delayed chemotherapy-induced nausea and vomiting: Pooled data from 2 randomised, double-blind, placebo controlled trials. Eur J Cancer, 41, 1278-85.

Warr DG, Hesketh PJ, Gralla RJ, et al (2005b). Efficacy and tolerability of aprepitant for the prevention of chemotherapyinduced nausea and vomiting in patients with breast cancer after moderately emetogenic chemotherapy. J Clin Oncol, 23, 2822-30.

Yeo W, Mo FK, Suen JJ, et al (2009). A randomized study of aprepitant, ondansetron and dexamethasone for chemotherapy-induced nausea and vomiting in Chinese breast cancer patients receiving moderately emetogenic chemotherapy. Breast Cancer Res Treat, 113, 529-35. 